\title{
A Modified Method for Harmonic Elimination
}

\author{
Sandeep V. Rode and Siddharth A. Ladhake
}

\begin{abstract}
-a modified filter strategy for harmonic elimination is introduced in this paper. The proposed approach eliminates all harmonics component from the signal (current or voltage) and it requires only knowledge of fundamental frequency of the signal. The proposed filter eliminates undesirable harmonics from periodic signal using adaptive algorithm. Adaptation process adjusts weights to exactly match amplitude and phase of fundamental frequency component and the outputs of the filter is a harmonics replica and are subtracted from the original periodic signal waveform to eliminate them. The bipolar waveforms are roughly analyzed and considered case of square wave pattern which contain all odd harmonics. The simulation results show that the method can effectively eliminate undesirable harmonics and result in low(less than two percentage) total harmonic distortion (THD).
\end{abstract}

Index Terms-Harmonics Elimination, LMS Algorithm, Selective harmonic elimination, Square wave generator

\section{INTRODUCTION}

In recent decades, the growing and widespread use of electronic equipment by different segment of society is perceptible. This equipment present itself as nonlinear impedances to its supplying electrical systems and generates harmonics with well-known adverse effects, such as low power factor, electromagnetic interface, voltage distortion, etc..A harmonic is a signal or wave whose frequency is an integral (whole-number) multiple of the frequency of some reference signal or wave [1]. For a signal whose fundamental frequency is $f$, the second harmonic has a frequency $2 \mathrm{f}$; the third harmonic has frequency of $3 \mathrm{f}$, and so on. Signal occurring at frequencies of $2 f, 4 f$, $6 f$, etc. are called even harmonics; the frequencies $3 \mathrm{f}, 5 \mathrm{f}, 7 \mathrm{f}$ etc. are called odd harmonics. If all the energy in a signal is contained at the fundamental frequency, then that signal is a perfect sin wave. If the signal is not a perfect sine wave, then some energy is contained in the harmonics. Examples are square wave, saw tooth wave and triangular wave.

It is well know that any periodic waveform such as that mentioned previously can be represented by Fourier series, an infinite sequence of sine and cosine waves, at the fundamental frequency of the waveforms and its harmonics. These harmonics can cause trouble in several areas particularly in motors and sensitive application. The coefficients of the Fourier series are computed with a pair of integral's that produces the coefficients of the sine and cosine terms in series. For a signal $\mathrm{f}(\mathrm{x})$ with a zero $\mathrm{dc}$

Manuscript received December 22, 2010; revised April 22, 2011.

S. V. Rode is with the Sipna's college of Engineering and Technology, Amravati, Maharashtra INDIA. (e-mail: sandeeprode30@yahoo.com)

S. A. Ladhake is with the Sipna's college of Engineering and Technology, Amravati, Maharashtra INDIA. component, the integrals are

$$
\begin{array}{ll}
A n=(1 / \pi) \int f(x) \cos (n x) d x & n>0 \\
B n=(1 / \pi) \int f(x) \sin (n x) d x & n>0
\end{array}
$$

where the An and Bn terms are the coefficients of the cosine and sine terms, respectively, in the series. The Fourier series is then:

$$
\begin{gathered}
f(x)=A_{1} \cos x+A_{2} \cos 2 x+A_{3} \cos 3 x-----+B_{1} \sin x \\
+B_{2} \sin 2 x+B_{3} \sin 3 x+(1)
\end{gathered}
$$

In conventional square wave have both half-wave symmetry and quarter-wave symmetry, integration is required only over one-quarter of the waveform, and further that only the sine terms and odd harmonics are required. Thus, the integral used to compute the coefficients for the conventional square wave becomes

$$
\begin{aligned}
& \mathrm{Bn}=(4 / \pi) \quad \mathrm{f}(\mathrm{x}) \sin (\mathrm{nx}) \mathrm{dx} \\
& (4 / \mathrm{n} \pi) \quad \text { for odd values of } \mathrm{n} \text { only. }
\end{aligned}
$$

The series is then $(4 / \pi) \sin (\mathrm{x})+(4 / 3 \pi) \sin (3 \mathrm{x})+(4 / 5 \pi$ $\sin (5 x)+(2)$

The standard measure for distortion is Total Harmonic Distortion (THD).Numerical evaluation of the coefficient for the square wave indicates that if the square wave is to be considered a sine wave with distortion, the THD is in the range of $45 \%$. The third harmonic, the hardest to filter out, is one-third the magnitude of the fundamental.

In paper [2] a superior scheme for producing nearly sinusoidal output waveforms using the modified Walsh function harmonic elimination method is described. A modulation based method for generating pulse waveforms with selective harmonics elimination is proposed in [3]. Another method to eliminate harmonics in single-phase PWM inverter is highlighted in [4].The main challenge is associated with elimination of all harmonics.

This manuscript proposes an approach for harmonic elimination based an adaptive filter technology is developed. The task is accomplished by generating harmonics replica using fundamental frequency of composite signal. The output of adaptive filter is a harmonics replica and is subtracted from the composite signal of fundamental frequency to eliminate them and the total harmonic distortion (THD) is greatly reduced. The weights of filter are adjusted on-line by using LMS adaptive filtering algorithm. The above course is used as a basis for the development of adaptive harmonics elimination algorithm. From the comparison result, it is seen clearly that this method has better harmonic elimination efficiency and faster weight convergence.

\section{ANALYZING HARMONICS}

Harmonics are AC voltages and currents with frequencies that are integer multiples of fundamental frequency. On a $50 \mathrm{~Hz}$ system, this could include $2^{\text {nd }}$ order harmonic $100 \mathrm{~Hz}$, $3 \mathrm{rd}$ order harmonic $(150 \mathrm{~Hz})$, 4th order harmonics $(200 \mathrm{~Hz})$, and so on. 
The flattened and dimpled sinusoid in Fig. 1 has the mathematical equation $y=2 \sin (2 \pi 50)+0.5 \sin (3 * 2 \pi 50)$. This means a $50 \mathrm{~Hz}$ sinusoid(the fundamental frequency) added to a second sinusoid with a frequency three times greater than the fundamental $(150 \mathrm{~Hz})$ and an amplitude $1 / 4(0.5$ times $)$ of the fundamental frequency.

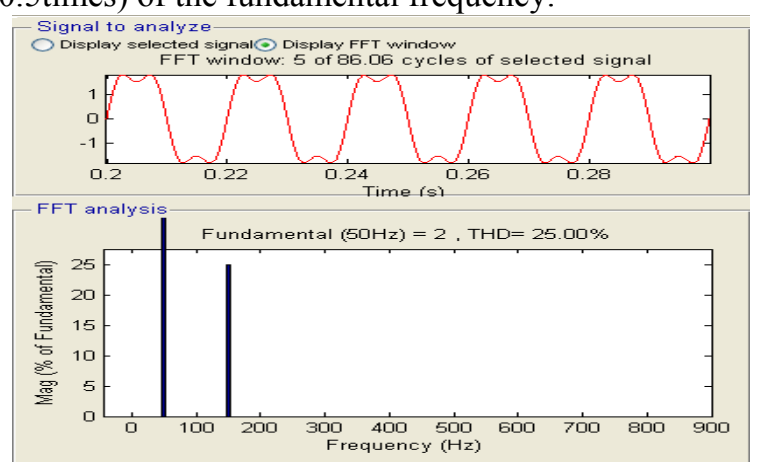

Fig.1: flattened and dimpled sinusoid waveform

Similarly the peaky sinusoid in Fig. 2 has the mathematical equation $y=2 \sin (2 \pi 50)-0.5 \sin (3 * 2 \pi 50)$. This waveform has the same composition as the first waveform except the third harmonics component is out of phase with the fundamental frequency, as indicated by the negative sign preceding the " $0.5 \sin (3 * 2 \pi 50)$ "term. The waveform in Fig. 3 contains third, fifth, seven, nine and eleven harmonics in phase with the fundamental frequency. The waveform in Fig. 4 contains several other harmonics in addition to the second harmonic some are in phase with the fundamental frequency and others out of phase.

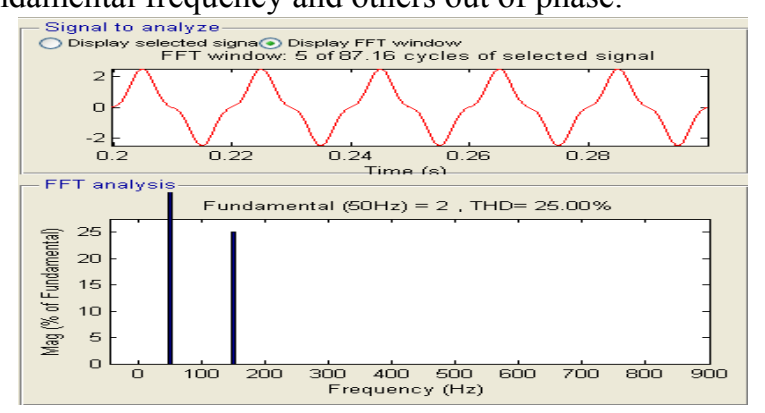

Fig. 2: peaky sinusoid waveform

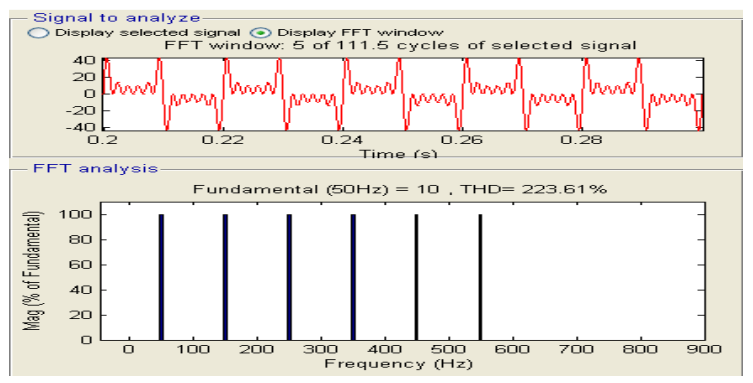

Fig. 3: The wave contains third, fifth, seven, nine and eleven harmonics

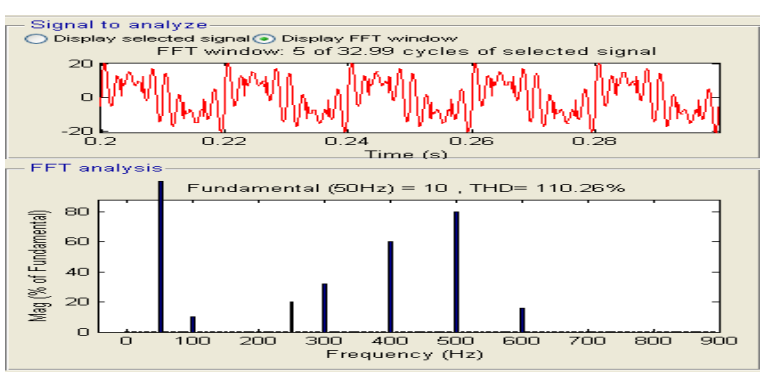

Fig.4. contain several other harmonics in addition to the second harmonic

\section{Filtering Using RC AND LC FiLTER}

As the harmonic spectrum becomes richer in harmonics the waveform takes on more complex appearance, indicating more deviation from the ideal sinusoid. A rich harmonic spectrum may completely obscure the fundamental frequency sinusoid, making a sine wave unrecognizable. When the magnitude and order of harmonics are known, reconstructing distorted waveform is simple. Decomposing a distorted waveform into its harmonic component is considerably more difficult. This process requires, Fourier analysis, which involves a fair amount of calculus. However, electronic equipment has been developed to perform this analysis on a real time basis. One of the strategies is to reduce the magnitude of the harmonic waveform, usually by filtering. Simple two RC pair low pass filter can be used for filtering the harmonics as shown in Fig.5, Resultant input and output of RC filter are shown in Fig. 6 output contain fundamental frequency component of amplitude $0.005598 \mathrm{~V}$ instead of 12.73 and output is load dependent.

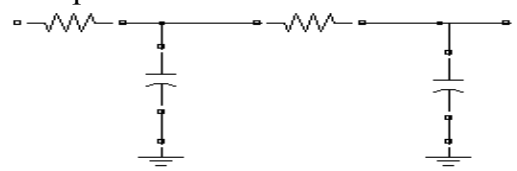

Fig.5: RC filter

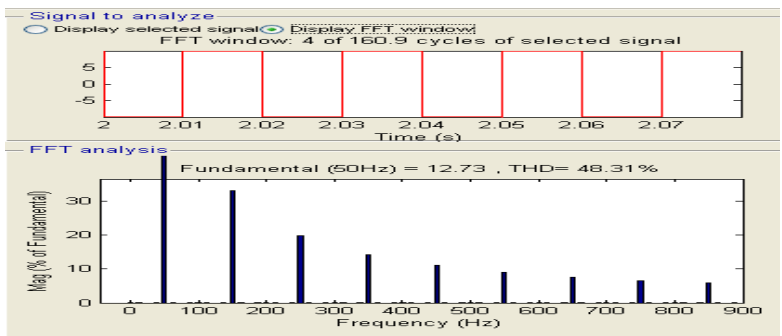

(a)

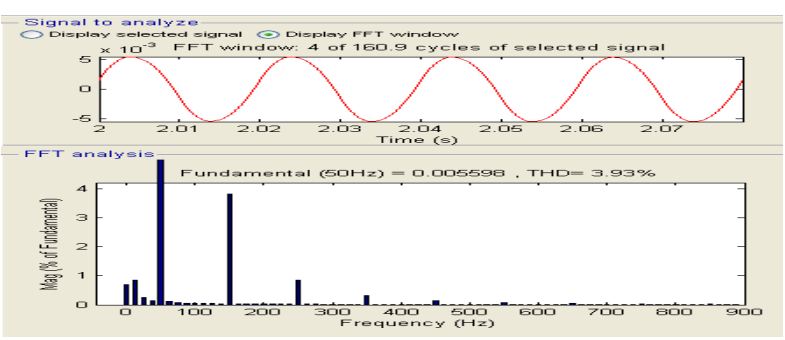

(b)

Fig. 6: Performance of RC filter:

(a) Output waveform without filter for $10 \mathrm{~V}, 50 \mathrm{~Hz}$

(b) Output waveform with filter for $10 \mathrm{~V}, 50 \mathrm{~Hz}$

LC low pass filter can be used for filtering the harmonics as shown in Fig.7, Resultant output of LC filter shown in Fig 8 contain fundamental frequency component of amplitude 12.42 instead of 12.73 and output is load dependent and unstable. The other method is to use system components that can handle the harmonics more effectively, such as $\mathrm{K}$-factor transformers. Harmonic filters can be constructed by adding an inductance (L) in series with a power factor correction capacitor(C). The series L-C circuit can be tuned for a frequency close to that of the troublesome harmonic, which is often the $5^{\text {th }}$ by tuning the filter in this way; you can attenuate the unwanted harmonic. This can be a very cost effective means of reducing 
harmonics. Because of the computation difficulty of the resultant method to eliminate all harmonics an approach is propose using LMS Algorithm.

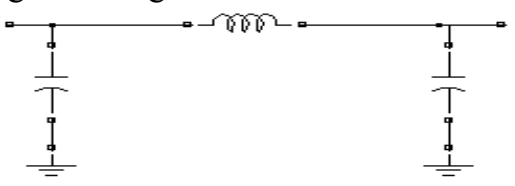

Fig.7: LC filter

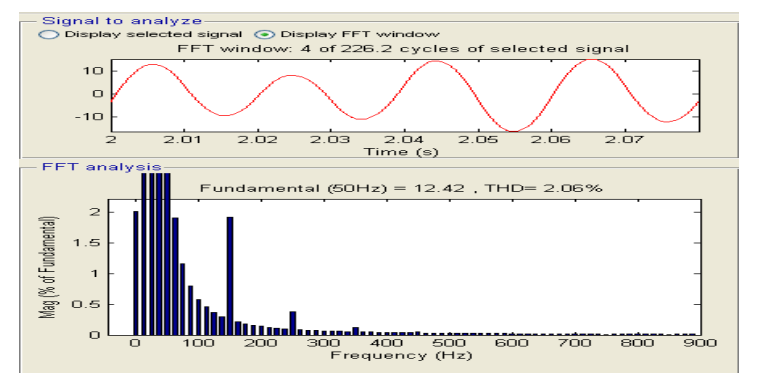

Fig. 8: Performance of LC filter: Output waveform with filter for 10V, $50 \mathrm{~Hz}$

\section{Propsed Harmonic Elimination Filter}

Elimination of an undesirable harmonic component from a signal can be done by proposed approach. The circuit consists of a summing point and least mean square (LMS) adaptive Algorithm. It operates in the following way:

a) The signal of frequency $F$ eliminated from composite signal. Elimination of fundamental frequency $\mathrm{F}$ is obtained using LMS algorithm.

b) Adaptation process adjusts weights to exactly match amplitude and phase of fundamental frequency component $\mathrm{F}$.

c) The signal created by a filter circuit is subtracted from the primary input, such that the output harmonics are cancelled leaving the desired fundamental frequency signal alone.

The LMS adaptation algorithm as developed in [5] will discussed as Fig. 9 is a block diagram of system identification using adaptive filtering. The objective is to change (adapt) the coefficients of an FIR filter, W, to match as closely as possible the response of an unknown system, $\mathrm{H}$. The unknown system and the adapting filter process the same input signal $x[n]$ and have outputs $d[n]$ (also referred to as the desired signal) and $\mathrm{y}[\mathrm{n}]$.

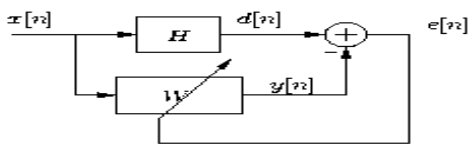

Fig.9: System identification block diagram

Gradient-descent adaptation: The adaptive filter, $W$, is adapted using the least mean-square algorithm, which is the most widely used adaptive filtering algorithm. First the error signal, $e[n]$, is computed as $e[n]=d[n]-y[n]$, which measures the difference between the output of the adaptive filter and the output of the unknown system. On the basis of this measure, the adaptive filter will change its coefficients in an attempt to reduce the error. The coefficient update relation is a function of the error signal squared and is given by

$$
\mathrm{hn}+1[\mathrm{i}]=\mathrm{hn}[\mathrm{i}]+\mu / 2\left\{-\left[\mathrm{a} / \mathrm{a} \mathrm{hn}[\mathrm{i}]\left((|\mathrm{e}|)^{2}\right)\right]\right\}
$$

The term inside the parentheses represents the gradient of the squared-error with respect to the $i^{\text {th }}$ coefficient. The gradient is a vector pointing in the direction of the change in filter coefficients that will cause the greatest increase in the error signal. Because the goal is to minimize the error, however, equation 3 updates the filter coefficients in the direction opposite the gradient; that is why the gradient term is negated. The constant $\mu$ is a step-size, which controls the amount of gradient information used to update each coefficient. After repeatedly adjusting each coefficient in the direction opposite to the gradient of the error, the adaptive filter should converge; that is, the difference between the unknown and adaptive systems should get smaller and smaller. The step-size $\mu$ directly affects how quickly the adaptive filter will converge toward the unknown system. If $\mu$ is very small, then the coefficients change only a small amount at each update, and the filter converges slowly. With a larger step-size, more gradient information is included in each update, and the filter converges more quickly; however, when the step-size is too large, the coefficients may change too quickly and the filter will diverge. (It is possible in some cases to determine analytically the largest value of $\mu$ ensuring convergence.)

The LMS reference design has the two main functional blocks: the FIR (or IIR) Filter and the LMS Algorithm. The FIR filter is implemented serially using a multiplier and an adder with a feedback as shown in the high level schematic from Fig.10. The FIR result is normalized to minimize saturation [6].

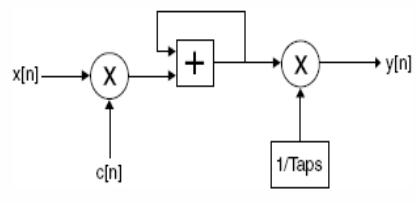

Fig.10: FIR filter

The LMS algorithm iteratively updates the coefficient and feeds it to the FIR filter. The FIR filter than uses the coefficient $\mathrm{c}(\mathrm{n})$ along with the input reference signal $\mathrm{x}(\mathrm{n})$ to generate the output $y(n)$. The output $y(n)$ is then subtracted from the desired signal $d(n)$ to generate an error, which is used by the LMS algorithm to compute the next set of coefficients.

\section{Simulation AND EXPERIMENTAL RESUlts}

Generally, square wave output inverter available in market is simple in design and low cost. Such inverter has the least desirable output waveform type; a square wave, [7] is sort of a "flattened-out" version of a sine wave and have some disadvantages. For starters, the peak voltage of a square wave is substantially lower than the peak voltage of a sine wave. In addition, a square wave contains many higher frequencies as well, called harmonics, which can cause buzzing or other problems.

A modified approach is proposed in this paper to eliminate all harmonics from square wave waveform. A square wave output waveform which contain many odd harmonics. First, a fundamental frequency of square wave is 
applied to LMS algorithm and LMS adaptive filter gives output of harmonics replica present in square wave output wave. Next, harmonics replica is subtracted from square wave output. Therefore, the output voltage wave is sinusoidal waveform of fundamental frequency. Input and output waveforms are shown in Fig. 11 using MATLAB/Simulink. As compare to RC or LC filter LMS algorithm gives better response: low THD (1.36\%), stable output and load independent. Table-I gives the performance parameters at various load conditions.

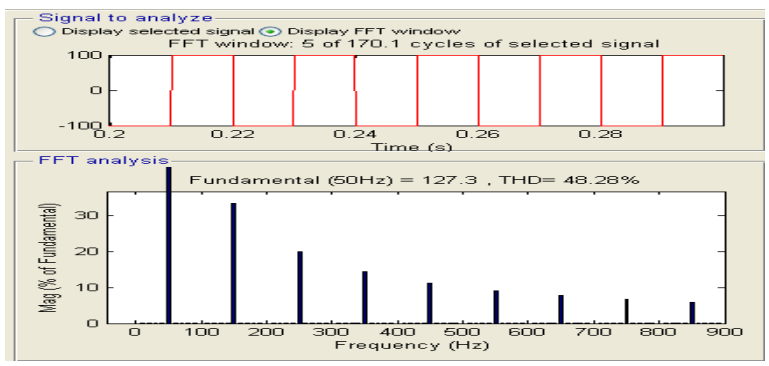

(a)

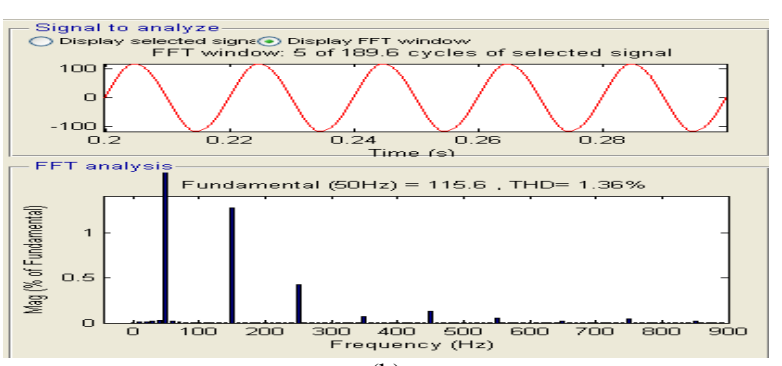

(b)

Fig.11: Performance of the proposed scheme.

(a) Output waveform without filter for $100 \mathrm{~V}, 50 \mathrm{~Hz}$

(b) Output waveform with filter for $100 \mathrm{~V}, 50 \mathrm{~Hz}$

TABLE I. PERFormance PARAMETER AT VARIOUS LOAD CONDITIONS

\begin{tabular}{|c|c|c|}
\hline Filter & THD & Output response \\
\hline RC & $3.93 \%$ & Load dependent \\
\hline LC & $2.06 \%$ & Load dependent \\
\hline LMS & $1.36 \%$ & Load independent \\
\hline
\end{tabular}

\section{CONCLUSION}

A modified method has been proposed and developed to eliminate undesirable harmonics from composite signal using LMS algorithm. The simulation results show that the proposed filter can be used to eliminate higher order harmonics effectively. The simulation result show that the method can effectively eliminate all odd harmonics from square wave and sine wave is produced with total harmonic distortion less than two percentages. The advantages of this approach are simple design, low THD and cost effective.

\section{REFERENCES}

[1] Cyril W. Lander, Power Electronics, Singapore, McGraw-HILL. International limited, 1993.pp 295-333.

[2] Tsorng-Juu Liang and Robert M O'Connell , "Inverter Harmonic reduction using Walsh function harmonic elimination method" IEEE Trans.Power Electron.,vol 12 no.6,pp.971-982 Nov.1997

[3] ]Brett M. Nee, Jason R. Wells, Xing Geng, Patrick L. Chapman and Philip T. Krein, "Modulation-Based Harmonic Elimination," IEEE Trans. Power Electron. vol. 22 no.1, pp.336-339, Jan 2007.

[4] Keliang Zhou,Kay-Soon Low Danwei Wang, "Zero-Phase OddHarmonic Repetitive Controller for a Single-Phase PWM Inverter," IEEE Trans. Power Electron. vol. 21 no.1, pp.193-200, Jan. 2006.

[5] B. Window and S. D. Stearns, Adaptive signal processing. Englewood cliffs, NJ: Prentice-Hall, 1985.

[6] Lattice Semiconductor Corporation, "LMS Adaptive Filter", Reference Design RD1031,December 2006

[7] Ashfaq Ahmed, Power Electronics for Technology, Singapore, Pearson Education Pte. Ltd., 2003. pp. 304-312.

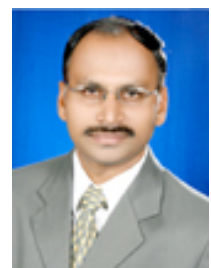

Sandeep Vinayakrao Rode, received master's degree in 2004 in Electronics Engineering from Sant Gadge baba Amravati University, Amravati, India. He has 12 years of teaching experience and presently working as Assistant professor in Department of Electronics and Telecommunication at sipna's college of Engineering and Technology, Amravati(India).His main research interest include signal processing, power Electronics etc. and doing $\mathrm{PhD}$ in signal processing .He is a member of IEEE,IETE,IE and ISTE. He has published nine papers in National and International Journal and conferences.

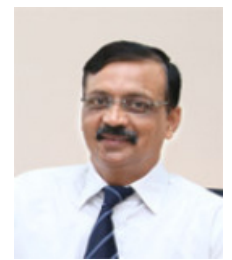

Siddharth A. Ladhake, received master's degree in 1990 and $\mathrm{PhD}$ in 2004 in Electronics Engineering from Sant Gadge baba Amravati University, Amravati, India. He has 27 years of teaching experience and presently working as Principal at sipna's college of Engineering and Technology, Amravati(India).Previously he was holding a post of professor from 1999 to 2005 at Prof. Ram meghe institutes of Engineering and research, Badnera,(India).His main research interest include signal processing, image processing, code conversion Multivalued logic etc. $\mathrm{He}$ isa member of IEEE, IETE, IE and ISTE. He has published 37 papers in National and International Journal and conferences. 